PROCEEDINGS OF THE

AMERICAN MATHEMATICAL SOCIETY

Volume 134, Number 11, November 2006, Pages 3181-3189

S 0002-9939(06)08331-6

Article electronically published on June 1, 2006

\title{
POISSON INTEGRALS AND NONTANGENTIAL LIMITS
}

\author{
VICTOR L. SHAPIRO
}

(Communicated by Juha M. Heinonen)

\begin{abstract}
A new result is established for nontangential limits of the Poisson integral of an $f \in L^{p}\left(\mathbf{R}^{N}\right)$ for $N \geq 2$. This is accomplished by showing for $N=2, \exists f$ such that the $\sigma$-set of $f$ strictly contains the Lebesgue set of $f$. A similar theorem is also proved for Gauss-Weierstrass integrals, giving a new result for solutions of the heat equation.
\end{abstract}

\section{INTRODUCTION}

We shall operate in real $N$-dimensional Euclidean space, $\mathbf{R}^{N}, N \geq 1$, and use the following notation:

$$
\begin{aligned}
x & =\left(x_{1}, \ldots, x_{N}\right), \quad y=\left(y_{1}, \ldots, y_{N}\right), \\
\alpha x+\beta y & =\left(\alpha x_{1}+\beta y_{1}, \ldots, \alpha x_{N}+\beta y_{N}\right), \\
x \cdot y & =x_{1} y_{1}+\ldots+x_{N} y_{N}, \quad|x|=(x \cdot x)^{\frac{1}{2}} .
\end{aligned}
$$

For $t>0$ and $x \in \mathbf{R}^{N}$, we define the Poisson kernel, $P(x, t)$, to be

$$
P(x, t)=a_{N} t\left[t^{2}+|x|^{2}\right]^{-(N+1) / 2} \text { where } a_{N}=\frac{\Gamma\left(\frac{N+1}{2}\right)}{\pi^{(N+1) / 2}} .
$$

As is well known (e.g., see [SW, p. 9])

$$
\int_{\mathbf{R}^{N}} P(x, t) d x=1 \text { for } t>0 .
$$

For $f \in L^{p}\left(\mathbf{R}^{N}\right), 1 \leq p \leq \infty$, we set

$$
u(x, t)=\int_{\mathbf{R}^{N}} f(x+y) P(y, t) d y \text { for } t>0,
$$

and refer to $u(x, t)$ as the Poisson integral of $f$.

In this paper, we will be concerned with nontangential limits of $u(x, t)$. With $x_{0} \in \mathbf{R}^{N}$ and $\gamma>0$, let $\mathcal{C}_{\gamma}\left(x_{0}\right)$ stand for the cone in $\mathbf{R}_{+}^{N+1}$ with vertex $\left(x_{0}, 0\right)$ given as follows:

$$
\mathcal{C}_{\gamma}\left(x_{0}\right)=\left\{(x, t): t>0 \text { and } \frac{t}{\left|x-x_{0}\right|} \geq \gamma\right\}
$$

Received by the editors September 24, 2004 and, in revised form, April 26, 2005.

2000 Mathematics Subject Classification. Primary 31B25, 35K20; Secondary 35J05, 35K05.

Key words and phrases. Nontangential limit, Poisson integral, Gauss-Weierstrass integral. 
We say that $u(x, t)$ has the nontangential limit $l$ at $x_{0}$ provided the following prevails:

$$
\forall \gamma>0, \quad \lim _{(x, t) \rightarrow\left(x_{0}, 0\right)} u(x, t)=l \quad(x, t) \text { in } \mathcal{C}_{\gamma}\left(x_{0}\right) .
$$

We shall write (1.5) as

$$
\lim _{(x, t) \rightarrow\left(x_{0}, 0\right)} u(x, t)=l \quad \text { nontangentially. }
$$

In [SW, p. 62], it is shown that if $x_{0}$ is in the Lebesgue set of $f$, then $u(x, t)$ has the nontangential limit $f\left(x_{0}\right)$ at $x_{0}$. It is the purpose of this paper to improve upon this result, and the one which we present here appears to be new for dimension $N \geq 2$. In dimension $N=1$, our result is equivalent to the nontangential limit theorem given in [Z, p. 101]. In $\S 3$, we will give an example in the plane (which is easily extendable to one in higher dimensions) to show that our theorem is a true improvement over the result in [SW, p. 62].

In order to do all this, we introduce the $\sigma$-set of $f$. With $B(x, r)$ designating the open ball with center $x$ and radius $r$, we say $x_{0}$ is in the $\sigma$-set of $f$ provided the following holds: $\forall \varepsilon>0, \exists \delta>0$ such that

$$
\left|x-x_{0}\right|<\delta \text { and } r<\delta \Longrightarrow\left|\int_{B(x, r)}\left(f(y)-f\left(x_{0}\right)\right) d y\right|<\varepsilon\left(\left|x-x_{0}\right|+r\right)^{N} .
$$

We intend to prove the following theorem regarding the $\sigma$-set of $f$ and nontangential limits.

Theorem 1. With $f \in L^{p}\left(\mathbf{R}^{N}\right), 1 \leq p \leq \infty$, set

$$
u(x, t)=\int_{\mathbf{R}^{N}} f(x+y) P(y, t) d y \quad \text { for } \quad t>0 .
$$

Then if $x_{0}$ is in the $\sigma-$ set of $f$,

$$
\lim _{(x, t) \rightarrow\left(x_{0}, 0\right)} u(x, t)=f\left(x_{0}\right) \quad \text { nontangentially. }
$$

We recall that " $x_{0}$ is in the Lebesgue set of $f$ " means that

$$
\lim _{r \rightarrow 0} r^{-N} \int_{B\left(x_{0}, r\right)}\left|f(y)-f\left(x_{0}\right)\right| d y=0 .
$$

Observing that $B(x, r) \subset B\left(x_{0},\left|x-x_{0}\right|+r\right)$, we see from this last limit and (1.6) that $x_{0}$ in the Lebesgue set of $f$ implies that $x_{0}$ is in the $\sigma$-set of $f$. Hence the theorem above is an improvement of the theorem given in [SW] p. 62] concerning nontangential limits of the Poisson integral.

Likewise, it is easy to see that in dimension $N=1, x_{0}$ in the $\sigma$-set of $f$ is equivalent to the fact that $F(x)=\int f$ has a finite derivative $f\left(x_{0}\right)$ at $x_{0}$. Hence, for $N=1$, our theorem above is equivalent to the result given in [Z, p. 61].

In $\S 4$ of this paper, we will prove a nontangential limit theorem for GaussWeierstrass integrals, thus giving a new result for solutions of the heat equation.

\section{Proof of Theorem 1}

Letting $\overline{0}=(0, \ldots, 0)$ represent the point in $\mathbf{R}^{N}$ all of whose components are zero, we see with no loss in generality that we can assume from the start that $x_{0}=\overline{0}$. 
Next, let $\gamma>0$ be given, and assume that

$$
\left\{\left(x_{n}, t_{n}\right)\right\}_{n=1}^{\infty} \subset \mathcal{C}_{\gamma}(\overline{0}) \text { and } \lim _{n \rightarrow \infty}\left(x_{n}, t_{n}\right)=(\overline{0}, 0) \text {. }
$$

The theorem will be established if we can show the following: Let $\varepsilon>0$ be given. Then,

$$
\limsup _{n \rightarrow \infty} \frac{\left|u\left(x_{n}, t_{n}\right)-f(\overline{0})\right|}{a_{N}} \leq 2(N+1)\left(\gamma^{-1}+1\right)^{N} \varepsilon .
$$

To show that (2.2) is true, we first observe from (1.1)-(1.3) that

$$
\frac{u\left(x_{n}, t_{n}\right)-f(\overline{0})}{a_{N}}=t_{n} \int_{\mathbf{R}^{N}} \frac{\left[f\left(x_{n}+y\right)-f(\overline{0})\right]}{\left[t_{n}^{2}+|y|^{2}\right]^{(N+1) / 2}} d y .
$$

On setting

$$
F_{n}(r)=\int_{B\left(x_{n}, r\right)}[f(y)-f(\overline{0})] d y,
$$

and using the fact that $\overline{0}$ is in the $\sigma$-set of $f$, we invoke (1.6) and choose $\delta$ so that

$$
\left|F_{n}(r)\right| \leq \varepsilon\left(\left|x_{n}\right|+r\right)^{N} \quad \text { for } \quad\left|x_{n}\right|<\delta \text { and } r<\delta .
$$

Next, we see from Holder's inequality that there exists a constant $K>0$ such that

$$
\int_{\mathbf{R}^{N}-B(\overline{0}, \delta)} \frac{\left|f\left(x_{n}+y\right)-f(\overline{0})\right|}{|y|^{N+1}} d y \leq K \quad \forall n .
$$

Hence, it follows from (2.3) and this last inequality that (2.2) will be established if we can succeed in showing

$$
\limsup _{n \rightarrow \infty}\left|t_{n} \int_{B(\overline{0}, \delta)} \frac{\left[f\left(x_{n}+y\right)-f(\overline{0})\right]}{\left[t_{n}^{2}+|y|^{2}\right]^{(N+1) / 2}} d y\right| \leq 2(N+1)\left(\gamma^{-1}+1\right)^{N} \varepsilon .
$$

From (2.4), we see that

$$
F_{n}(r)=\int_{B(\overline{0}, r)}\left[f\left(x_{n}+y\right)-f(\overline{0})\right] d y .
$$

Consequently, $F_{n}(r)$ is absolutely continuous on the interval $(0, \delta)$ with $\frac{d F_{n}(r)}{d r}$ existing almost everywhere in $(0, \delta)$ and also in $L^{1}(0, \delta)$. Therefore, the integral in (2.6) is equal to

$$
\int_{0}^{\delta} \frac{d F_{n}(r)}{d r}\left(t_{n}^{2}+r^{2}\right)^{-(N+1) / 2} d r
$$

We conclude, after integrating by parts, that the inequality in (2.6) will be established if we show

$$
\limsup _{n \rightarrow \infty}\left|t_{n} \int_{0}^{\delta} r F_{n}(r)\left(t_{n}^{2}+r^{2}\right)^{-(N+3) / 2} d r\right| \leq 2\left(\gamma^{-1}+1\right)^{N} \varepsilon .
$$

Next, we observe that $\int_{0}^{\delta}=\int_{0}^{t_{n}}+\int_{t_{n}}^{\delta}$. We shall deal with each of these cases separately and show

$$
\limsup _{n \rightarrow \infty}\left|t_{n} \int_{0}^{t_{n}} r F_{n}(r)\left(t_{n}^{2}+r^{2}\right)^{-(N+3) / 2} d r\right| \leq\left(\gamma^{-1}+1\right)^{N} \varepsilon
$$


and

$$
\limsup _{n \rightarrow \infty}\left|t_{n} \int_{t_{n}}^{\delta} r F_{n}(r)\left(t_{n}^{2}+r^{2}\right)^{-(N+3) / 2} d r\right| \leq\left(\gamma^{-1}+1\right)^{N} \varepsilon .
$$

If the inequalities in (2.8) and (2.9) are established, then the inequality in (2.7) follows. So to complete the proof of the theorem, it remains to show that the inequalities in (2.8) and (2.9) are valid.

We proceed with the situation in (2.8). For this case, $t_{n}<\delta$ and $0<r<t_{n}$. Also, from (2.1) and(1.4), $\left|x_{n}\right| \leq \gamma^{-1} t_{n}$. Consequently, we see from (2.5) for this case

$$
\left|F_{n}(r)\right| \leq \varepsilon\left(\left|x_{n}\right|+r\right)^{N} \leq \varepsilon\left(\gamma^{-1} t_{n}+t_{n}\right)^{N}
$$

Therefore,

$$
\begin{aligned}
\left|\int_{0}^{t_{n}} r F_{n}(r)\left(t_{n}^{2}+r^{2}\right)^{-(N+3) / 2} d r\right| & \leq \varepsilon\left(\gamma^{-1}+1\right)^{N} t_{n}^{N} \int_{0}^{t_{n}} r\left(t_{n}^{2}+r^{2}\right)^{-(N+3) / 2} d r \\
& \leq \varepsilon\left(\gamma^{-1}+1\right)^{N} t_{n}^{N} \int_{0}^{t_{n}} r t_{n}^{-(N+3)} d r \\
& \leq \varepsilon\left(\gamma^{-1}+1\right)^{N} t_{n}^{-1} / 2
\end{aligned}
$$

and we conclude that the inequality in (2.8) does indeed hold.

So to complete the proof of the theorem, it remains to show that the inequality in (2.9) is valid. For this case, $t_{n}<r<\delta$, and from (2.1) and (1.4), we also see that $\left|x_{n}\right| \leq \gamma^{-1} t_{n} \leq \gamma^{-1} r$. Consequently, we infer from (2.5) that

$$
\left|F_{n}(r)\right| \leq \varepsilon\left(\left|x_{n}\right|+r\right)^{N} \leq \varepsilon\left(\gamma^{-1} r+r\right)^{N} .
$$

Therefore,

$$
\begin{aligned}
\left|\int_{t_{n}}^{\delta} r F_{n}(r)\left(t_{n}^{2}+r^{2}\right)^{-(N+3) / 2} d r\right| & \leq \varepsilon\left(\gamma^{-1}+1\right)^{N} \int_{t_{n}}^{\delta} r^{N+1}\left(t_{n}^{2}+r^{2}\right)^{-(N+3) / 2} d r \\
& \leq \varepsilon\left(\gamma^{-1}+1\right)^{N} \int_{t_{n}}^{\delta} r^{N+1} r^{-(N+3)} d r \\
& \leq \varepsilon\left(\gamma^{-1}+1\right)^{N} t_{n}^{-1},
\end{aligned}
$$

and we conclude that the inequality in (2.9) is indeed valid. The proof of the theorem is therefore complete.

\section{An eXAmple}

In this section, we give an example of an $f \in L^{p}\left(\mathbf{R}^{2}\right), 1 \leq p \leq \infty$, with $\overline{0}=(0,0)$ in the $\sigma$-set of $f$ and such that $\overline{0}$ is not in the Lebesgue set of $f$. This example is easily extendable to one in $L^{p}\left(\mathbf{R}^{N}\right), N \geq 3$. Hence, our theorem above is a true improvement over the one in SW, p. 62].

To exhibit our example, we first consider the function $h_{n}(s)$ defined on the interval $(n+1)^{-1} \leq s \leq n^{-1} \forall n \geq 1$. In order to this, we first introduce the five points $\left\{\xi_{j}^{n}\right\}_{j=0}^{4}$ which subdivide the interval $\left[(n+1)^{-1}, n^{-1}\right]$ into four equal intervals, namely

$$
\xi_{j}^{n}=\frac{1}{n+1}+\frac{j}{4 n(n+1)}, \quad j=0,1,2,3,4 .
$$


Next, we define $h_{n}(s)$ to be linear in each of the intervals $\left[\xi_{0}^{n}, \xi_{1}^{n}\right],\left[\xi_{1}^{n}, \xi_{3}^{n}\right]$, and $\left[\xi_{3}^{n}, \xi_{4}^{n}\right]$ with $h_{n}\left(\xi_{0}^{n}\right)=h_{n}\left(\xi_{2}^{n}\right)=h_{n}\left(\xi_{4}^{n}\right)=0$ and $h_{n}\left(\xi_{1}^{n}\right)=1$ and $h_{n}\left(\xi_{3}^{n}\right)=-1$. In other words,

$$
\begin{array}{rlrl}
h_{n}(s) & =4 n(n+1)\left(s-\xi_{0}^{n}\right) & & \text { for } \xi_{0}^{n} \leq s \leq \xi_{1}^{n} \\
& =4 n(n+1)\left(\xi_{2}^{n}-s\right) & \text { for } \xi_{1}^{n} \leq s \leq \xi_{3}^{n} \\
& =4 n(n+1)\left(s-\xi_{4}^{n}\right) & \text { for } \xi_{3}^{n} \leq s \leq \xi_{4}^{n} .
\end{array}
$$

We then define $g(s)$ on the half-open interval $(0,1]$ as follows:

$$
g(s)=h_{n}(s) \quad \text { for } \quad s \in\left[(n+1)^{-1}, n^{-1}\right], \quad n=1,2, \ldots,
$$

and then on $\mathbf{R}$ in the following manner:

$$
\begin{aligned}
g(s) & =0 \text { for } s=0 \text { and } s \geq 1 \\
& =-g(-s) \text { for } s \leq 0 .
\end{aligned}
$$

It is clear that $g(s)$ is uniformly bounded in $\mathbf{R}$ and continuous everywhere except $s=0$. However, if we define $G(s)=\int_{0}^{s} g(t) d t$ for $s \in \mathbf{R}$, we see that $G(0)=0$, that $G(s)$ is an even function, and that

$$
|G(s)| \leq[4 n(n+1)]^{-1} \quad \text { for } s \in\left[(n+1)^{-1}, n^{-1}\right], \quad n=1,2, \ldots .
$$

Therefore,

$$
\frac{|G(s)|}{s} \leq \frac{n+1}{4 n(n+1)} \leq s \quad \text { for } s \in\left[(n+1)^{-1}, n^{-1}\right], \quad n=1,2, \ldots,
$$

and consequently, this last inequality plus the fact that $G$ is an even function implies that

$$
\frac{|G(s)|}{|s|} \leq|s| \quad \text { for } 0<|s| \leq 1
$$

Since $G(0)=0$, we obtain from (3.3) that the derivative of $G$ exists at 0 with $\frac{d G}{d s}(0)=0$. But then from the definition of $G$ and the fact that $g(0)=0$, we have

$$
\frac{d G}{d s}(s)=g(s) \quad \forall s \in \mathbf{R} .
$$

Also, we see that

$$
\int_{0}^{s}|g(t)| d t \geq \sum_{k=n+1}^{\infty} \frac{1}{2 k(k+1)} \quad \text { for } s \in\left[(n+1)^{-1}, n^{-1}\right], \quad n=1,2, \ldots,
$$

and

$$
\sum_{k=n+1}^{\infty} \frac{1}{k(k+1)} \geq \sum_{k=n+2}^{\infty} \frac{1}{k^{2}} \geq \frac{1}{n+2} \geq \frac{s}{3} \text { for } s \in\left[(n+1)^{-1}, n^{-1}\right], \quad n=1,2, \ldots
$$

We conclude from these last two sets of inequalities that

$$
s^{-1} \int_{0}^{s}|g(t)| d t \geq 6^{-1} \quad \text { for } \quad 0<s \leq 1
$$

We now define the function for our example, namely $f\left(x_{1}, x_{2}\right) \in L^{p}\left(\mathbf{R}^{2}\right), 1 \leq$ $p \leq \infty$, as follows:

$$
\begin{aligned}
f\left(x_{1}, x_{2}\right) & =g\left(x_{1}\right) & & \text { for }\left(x_{1}^{2}+x_{2}^{2}\right)^{\frac{1}{2}} \leq 10 \\
& =0 & & \text { for }\left(x_{1}^{2}+x_{2}^{2}\right)^{\frac{1}{2}}>10 .
\end{aligned}
$$


Next, we let $S q(\overline{0}, r)$ be the square of side $2 r$ centered at $\overline{0}$, and observe that $S q(\overline{0}, r) \subset B(\overline{0}, 2 r)$. Therefore from (3.6), we have that for $0<r \leq 1$,

$$
\int_{B(\overline{0}, 2 r)}|f(x)| d x \geq \int_{S q(\overline{0}, r)}|f(x)| d x=\int_{-r}^{r}\left[\int_{-r}^{r}\left|g\left(x_{1}\right)\right| d x_{1}\right] d x_{2} \geq 4 r \int_{0}^{r}|g(s)| d s .
$$

Also, recalling that $g(0)=0$, we have that $f(\overline{0})=0$. Hence, we infer from this last set of inequalities and the inequality in (3.5) that

$$
r^{-2} \int_{B(\overline{0}, 2 r)}|f(x)-f(\overline{0})| d x \geq \frac{2}{3} \quad \text { for } 0<r \leq 1 .
$$

Consequently,

$$
\liminf _{r \rightarrow 0} r^{-2} \int_{B(\overline{0}, 2 r)}|f(x)-f(\overline{0})| d x \geq \frac{2}{3},
$$

and we conclude from (1.7) above that $\overline{0}$ is not in the Lebesgue set of $f$.

To complete our example, it remains to show that $\overline{0}$ is in the $\sigma$-set of $f$. To accomplish this, we set

$$
F\left(x_{1}, x_{2}\right)=G\left(x_{1}\right) \quad \text { for } \quad\left(x_{1}, x_{2}\right) \in \mathbf{R}^{2},
$$

and infer from (3.4) and (3.6) that $F$ has a total derivative at each point of $\mathbf{R}^{2}$, and furthermore, if $\left(x_{1}^{2}+x_{2}^{2}\right)^{\frac{1}{2}} \leq 2$, then

$$
\frac{\partial F}{\partial x_{1}}\left(x_{1}, x_{2}\right)=f\left(x_{1}, x_{2}\right) \text { and } \frac{\partial F}{\partial x_{2}}\left(x_{1}, x_{2}\right)=0 .
$$

We next invoke the version of Green's theorem given in [S, p. 262] and obtain that for $\left(x_{1}^{2}+x_{2}^{2}\right)^{\frac{1}{2}} \leq 1$ and $r \leq 1$,

$$
\int_{\partial B(x, r)} F\left(y_{1}, y_{2}\right) d y_{2}=\int_{B(x, r)}[f(y)-f(\overline{0})] d y,
$$

where we also have made use of the fact that $f(\overline{0})=0$.

Now,

$$
\begin{aligned}
\int_{\partial B(x, r)} F\left(y_{1}, y_{2}\right) d y_{2} & =r \int_{0}^{2 \pi} F\left(x_{1}+r \cos \theta, x_{2}+r \sin \theta\right) \cos \theta d \theta \\
& =r \int_{0}^{2 \pi} G\left(x_{1}+r \cos \theta\right) \cos \theta d \theta
\end{aligned}
$$

So from (3.9) and this last computation, we have that

$$
\left|\int_{B(x, r)}[f(y)-f(\overline{0})] d y\right| \leq r \int_{0}^{2 \pi}\left|G\left(x_{1}+r \cos \theta\right)\right| d \theta .
$$

Next, from the inequality in (3.3), we see that

$$
\left|G\left(x_{1}+r \cos \theta\right)\right| \leq\left|x_{1}+r \cos \theta\right|^{2} \quad \text { for } \quad\left|x_{1}+r \cos \theta\right| \leq 1 .
$$

Consequently, given $\varepsilon>0$ with $\varepsilon<1$, choose $\delta=\frac{\varepsilon}{2 \pi}$. Then, from (3.10) and (3.11), we see that

$$
\text { for }|x|<\delta \text { and } r<\delta, \quad\left|\int_{B(x, r)}[f(y)-f(\overline{0})] d y\right|<\varepsilon(|x|+r)^{2},
$$

and we conclude from (1.6) that $\overline{0}$ is indeed in the $\sigma$-set of $f$. Therefore, $\overline{0}$ is not in the Lebesgue set of $f$, but is in the $\sigma$-set of $f$, and our example is complete. 


\section{Gauss-Weierstrass integrals}

For $t>0$ and $x \in \mathbf{R}^{N}$, we define the Gauss-Weierstrass kernel, $W(x, t)$, to be

$$
W(x, t)=(4 \pi t)^{-N / 2} e^{-|x|^{2} / 4 t} .
$$

As is well known (e.g., see [SW, p. 9])

$$
\int_{\mathbf{R}^{N}} W(x, t) d x=1 \text { for } t>0 .
$$

For $f \in L^{p}\left(\mathbf{R}^{N}\right), 1 \leq p \leq \infty$, we set

$$
w(x, t)=\int_{\mathbf{R}^{N}} f(x+y) W(y, t) d y \text { for } t>0,
$$

and refer to $w(x, t)$ as the Gauss-Weierstrass integral of $f$. It turns out that for $w(x, t)$, we can establish a theorem similar to Theorem 1, our nontangential limit result for the Poisson integral. In particular, the following theorem holds.

Theorem 2. With $f \in L^{p}\left(\mathbf{R}^{N}\right), 1 \leq p \leq \infty$, set

$$
w(x, t)=\int_{\mathbf{R}^{N}} f(x+y) W(y, t) d y \quad \text { for } \quad t>0 .
$$

Then, if $x_{0}$ is in the $\sigma-$ set of $f$,

$$
\lim _{(x, t) \rightarrow\left(x_{0}, 0\right)} w(x, t)=f\left(x_{0}\right) \quad \text { nontangentially. }
$$

Proof. The proof proceeds in a manner similar to the proof of Theorem 1. Letting $\overline{0}=(0, \ldots, 0)$ represent the point in $\mathbf{R}^{N}$, all of whose components are zero, we see with no loss in generality that we can assume from the start that $x_{0}=\overline{0}$.

Next, let $\gamma>0$ be given, and assume that

$$
\left\{\left(x_{n}, t_{n}\right)\right\}_{n=1}^{\infty} \subset \mathcal{C}_{\gamma}(\overline{0}) \text { and } \lim _{n \rightarrow \infty}\left(x_{n}, t_{n}\right)=(\overline{0}, 0) \text {. }
$$

The theorem will be established if we can show the following: Let $\varepsilon>0$ be given. Then,

$$
\limsup _{n \rightarrow \infty}\left|w\left(x_{n}, t_{n}\right)-f(\overline{0})\right| \leq \eta_{N} \varepsilon,
$$

where $\eta_{N}=\frac{\left(\gamma^{-1}+1\right)^{N}}{2(4 \pi)^{N / 2}} \int_{0}^{\infty} r^{N+1} e^{-r^{2} / 4} d r$.

To show that (4.5) is true, we first observe from (4.1)-(4.3) that

$$
w\left(x_{n}, t_{n}\right)-f(\overline{0})=\left(4 \pi t_{n}\right)^{-N / 2} \int_{\mathbf{R}^{N}}\left[f\left(x_{n}+y\right)-f(\overline{0})\right] e^{-|y|^{2} / 4 t_{n}} d y .
$$

On setting

$$
F_{n}(r)=\int_{B\left(x_{n}, r\right)}[f(y)-f(\overline{0})] d y,
$$

and using the fact that $\overline{0}$ is in the $\sigma$-set of $f$, we invoke (1.6) and choose $\delta$ so that

$$
\left|F_{n}(r)\right| \leq \varepsilon\left(\left|x_{n}\right|+r\right)^{N} \quad \text { for } \quad\left|x_{n}\right|<\delta \text { and } r<\delta .
$$

Next, we see from Holder's inequality that

$$
\lim _{n \rightarrow \infty}\left(4 \pi t_{n}\right)^{-N / 2} \int_{\mathbf{R}^{N}-B(\overline{0}, \delta)}\left|f\left(x_{n}+y\right)-f(\overline{0})\right| e^{-|y|^{2} / 4 t_{n}} d y=0 .
$$


Hence, it follows from (4.6) and this last limit that (4.5) will be established if we can succeed in showing

$$
\limsup _{n \rightarrow \infty}\left|\left(4 \pi t_{n}\right)^{-N / 2} \int_{B(\overline{0}, \delta)}\left[f\left(x_{n}+y\right)-f(\overline{0})\right] e^{-|y|^{2} / 4 t_{n}} d y\right| \leq \eta_{N} \varepsilon .
$$

From (4.7), we see that

$$
F_{n}(r)=\int_{B(\overline{0}, r)}\left[f\left(x_{n}+y\right)-f(\overline{0})\right] d y .
$$

Consequently, $F_{n}(r)$ is absolutely continuous on the interval $(0, \delta)$, and we obtain as in the proof of Theorem 1 that the integral in (4.9) is equal to

$$
\int_{0}^{\delta} \frac{d F_{n}(r)}{d r} e^{-r^{2} / 4 t_{n}} d r
$$

We conclude, after integrating by parts, that the inequality in (4.9) will be established if we show

$$
\limsup _{n \rightarrow \infty}\left|\left(4 \pi t_{n}\right)^{-N / 2} \int_{0}^{\delta} F_{n}(r) \frac{r}{2 t_{n}} e^{-r^{2} / 4 t_{n}} d r\right| \leq \eta_{N} \varepsilon .
$$

Next, we observe that $\int_{0}^{\delta}=\int_{0}^{t_{n}}+\int_{t_{n}}^{\delta}$. We shall deal with each of these cases separately and show

$$
\limsup _{n \rightarrow \infty}\left|\left(4 \pi t_{n}\right)^{-N / 2} \int_{0}^{t_{n}} F_{n}(r) \frac{r}{2 t_{n}} e^{-r^{2} / 4 t_{n}} d r\right| \leq 0
$$

and

$$
\limsup _{n \rightarrow \infty}\left|\left(4 \pi t_{n}\right)^{-N / 2} \int_{t_{n}}^{\delta} F_{n}(r) \frac{r}{2 t_{n}} e^{-r^{2} / 4 t_{n}} d r\right| \leq \eta_{N} \varepsilon .
$$

Once the inequalities in (4.11) and (4.12) are established, then the inequality in (4.10) follows. So to complete the proof of the theorem, it remains to show that the inequalities in (4.11) and (4.12) are valid.

We proceed with the situation in (4.11). For this case, $t_{n}<\delta$ and $0<r<t_{n}$. Also, from (4.4) and(1.4), $\left|x_{n}\right| \leq \gamma^{-1} t_{n}$. Hence, we infer from (4.8), for this case

$$
\left|F_{n}(r)\right| \leq \varepsilon\left(\left|x_{n}\right|+r\right)^{N} \leq \varepsilon\left(\gamma^{-1} t_{n}+t_{n}\right)^{N}
$$

Therefore,

$$
\begin{aligned}
\left|\int_{0}^{t_{n}} F_{n}(r) \frac{r}{2 t_{n}} e^{-r^{2} / 4 t_{n}} d r\right| & \leq \varepsilon\left(\gamma^{-1}+1\right)^{N} t_{n}^{N}\left|\int_{0}^{t_{n}} \frac{r}{2 t_{n}} e^{-r^{2} / 4 t_{n}} d r\right| \\
& \leq \varepsilon\left(\gamma^{-1}+1\right)^{N} t_{n}^{N} \int_{0}^{t_{n}^{\frac{1}{2}}} r e^{-r^{2} / 4} d r .
\end{aligned}
$$

Consequently,

$$
\left|\left(4 \pi t_{n}\right)^{-N / 2} \int_{0}^{t_{n}} F_{n}(r) \frac{r}{2 t_{n}} e^{-r^{2} / 4 t_{n}} d r\right| \leq \varepsilon\left(\gamma^{-1}+1\right)^{N} t_{n}^{N / 2} \int_{0}^{t_{n}^{\frac{1}{2}}} r e^{-r^{2} / 4} d r
$$

and we conclude that the inequality in (4.11) does indeed hold. 
So to complete the proof of the theorem, it remains to show that the inequality in (4.12) is valid. For this case, $t_{n}<r<\delta$, and from (4.4) and (1.4), we also see that $\left|x_{n}\right| \leq \gamma^{-1} t_{n} \leq \gamma^{-1} r$. Hence, we infer from (4.8) that

$$
\left|F_{n}(r)\right| \leq \varepsilon\left(\left|x_{n}\right|+r\right)^{N} \leq \varepsilon\left(\gamma^{-1} r+r\right)^{N},
$$

and obtain from the definition of $\eta_{N}$ below (4.5) that

$$
\begin{aligned}
\left|\left(4 \pi t_{n}\right)^{-N / 2} \int_{t_{n}}^{\delta} F_{n}(r) \frac{r}{2 t_{n}} e^{-r^{2} / 4 t_{n}} d r\right| & \leq \varepsilon \frac{\left(\gamma^{-1}+1\right)^{N}}{2 t_{n}\left(4 \pi t_{n}\right)^{N / 2}} \int_{t_{n}}^{\delta} r^{N+1} e^{-r^{2} / 4 t_{n}} d r \\
& \leq \varepsilon \frac{\left(\gamma^{-1}+1\right)^{N}}{2(4 \pi)^{N / 2}} \int_{t_{n}^{\frac{1}{2}}}^{\infty} r^{N+1} e^{-r^{2} / 4} d r \\
& \leq \varepsilon \eta_{N} .
\end{aligned}
$$

So the inequality in (4.12) is indeed valid. The proof of the theorem is therefore complete.

\section{REFERENCES}

[S] V. L. Shapiro, On Green's Theorem, J. London Math. Soc. 32 (1957) pp.261-269. MR0089275 (19:644g)

[SW] E.M. Stein and G. Weiss, Introduction to Fourier Analysis on Euclidean Spaces, Princeton Univ. Press, Princeton, 1990. MR0304972 (46:4102)

[Z] A. Zygmund, Trigonometric Series, Vol. I, Cambridge Univ. Press, New York, 1959. MR0107776 (21:6498)

Department of Mathematics, University of California, Riverside, California 92521

E-mail address: shapiro@math.ucr.edu 